\title{
Yod
}

Revue des études hébraïques et juives

\section{Le concept de la religiosité dans l'œuvre d'Aharon Appelfeld à travers le personnage féminin}

On the Question of Religiosity in Appelfeld's Narration

$$
\text { לשאלת הרלגיוזיות ביצירתו של אהרן אפלפלד }
$$

\section{Masha Itzhaki}

\section{OpenEdition}

\section{Journals}

Édition électronique

URL : https://journals.openedition.org/yod/1428

DOI : $10.4000 /$ yod. 1428

ISSN : 2261-0200

\section{Éditeur}

INALCO

Édition imprimée

Date de publication : 1 octobre 2009

Pagination : 201-213

ISBN : 978-2-85831-178-1

ISSN : 0338-9316

\section{Référence électronique}

Masha Itzhaki, « Le concept de la religiosité dans l'œuvre d'Aharon Appelfeld à travers le personnage

féminin », Yod [En ligne], 14 | 2009, mis en ligne le 31 octobre 2011, consulté le 08 juillet 2021. URL: http://journals.openedition.org/yod/1428; DOI : https://doi.org/10.4000/yod.1428

Ce document a été généré automatiquement le 8 juillet 2021.

\section{(ब) $(1)$ ( $)$}

Yod est mis à disposition selon les termes de la Licence Creative Commons Attribution - Pas d'Utilisation Commerciale 4.0 International. 


\title{
Le concept de la religiosité dans l'œuvre d'Aharon Appelfeld à travers le personnage féminin
}

On the Question of Religiosity in Appelfeld's Narration

לשאלת הרלגיוזיות ביצירתו של אהרן אפלפלד

\author{
Masha Itzhaki
}

\section{Que veut dire le terme « religiosité »?}

1 Un dialogue long et complexe est mené dans la narration d'Appelfeld entre le Juif traditionnel, le Juif assimilé et le non-juif. Il est toujours lié à deux composants narratifs incontournables:

- À une présence féminine très forte, souvent liée au personnage de la mère disparue trop tôt et à sa substitution, très couramment non juive.

- Au voyage vers la montagne, les Carpates, qui incarne la quête de la nature et le besoin du retour aux sources et le lieu de la sainteté dans son état de pureté parfaite.

2 Ce dialogue qui est très souvent évoqué par l'auteur lui-même ainsi que par les chercheurs qui étudient son œuvre ${ }^{1}$, tourne autour la question de la religiosité : une expérience de nature mystique qui dépasse les institutions religieuses officielles, une sorte d'attirance vers la divinité par un lien direct qui se fait à travers la nature et qui touche au plus profond de l'âme humaine.

C'est ainsi que l'écrivain lui-même élabore ce sujet :

Le juif assimilé est semblable à une fleur qui pousserait hors du sol. Mais j'ai beaucoup écrit justement sur le non-juif qui comprend le judaïsme mieux que les juifs eux-mêmes.

Et puis :

Le non-juif, lui, sait qu'il y a des choses primordiales comme le service de Dieu, même s'il s'agit d'un service païen. Cela relève de l'essence même de l'homme. Le juif assimilé est trop rationaliste pour se sentir relié au mystère de la foi, du paganisme et de la nature. Trop rationaliste... ${ }^{2}$ 


\section{Le Hassidisme et Marie-Madeleine}

Le terme « rationaliste " n'est pas utilisé par hasard. Dans l'histoire du judaïsme, il se situe en opposition au Hassidisme, un mouvement juif populaire fondé en XVIII ${ }^{e}$ siècle en Europe de l'Est après la crise du faux messie Shabtaï Tzvi. Ce mouvement, dont certains centres étaient situés autour de Czernowitz ${ }^{3}$, la ville natale d'Appelfeld, constitue un contrepoids au judaïsme institutionnel, intellectuel et élitiste, existant dans les grandes écoles rabbiniques. Son objectif était de "démocratiser » la judaïcité, selon la formulation de Martin Buber, pour la rendre accessible non seulement aux Juifs aisés et instruits, habitants des grandes villes, mais aussi aux pauvres paysans qui n'avaient jamais fait d'études. Les Hassidim (ce terme est tiré de la racine hébraïque qui signifie à la fois grâce et piété) insistent particulièrement sur la communion joyeuse avec Dieu, en particulier par le chant et la danse. Le panthéisme et la contemplation constituent dans le Hassidisme les chemins spirituels qui permettent à chacun d'avoir un accès direct à la divinité. Selon cette doctrine, aucun lieu n'est vide de Dieu et l'énergie divine se répand aussi bien dans le monde matériel de la nature que dans celui du spirituel. C'est pourquoi on peut Le servir non seulement par les études approfondies de textes sacrés, mais aussi en satisfaisant des besoins corporels, eux aussi créés par Lui, à condition de le faire dans un esprit de sainteté et de ferveur ${ }^{4}$,

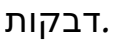

Ces deux principes, le panthéisme et la contemplation inspirent profondément l'idée de la religiosité, telle qu'elle est perçue par Appelfeld.

Vers la fin du roman La chambre de Mariana (פרחי האפלה) on trouve le passage suivant :

«À présent je vais m'asseoir et contempler. C'est comme ça que l'âme s'apaise. Tout ce que nous voyons et entendons est Dieu, disait ma grand-Mère, car Dieu réside partout, jusque dans le plus petit brin d'herbe». Par la suite Mariana évoque la beauté de la musique des Psaumes en disant: "Lis mon chéri, tu as une voix magnifique. Je ne comprends pas les chants, mais ils élèvent mon âme », et enfin elle donne le conseil suivant: "Méfie-toi de la mélancolie comme de la peste. ${ }^{6} "$

7 On peut sans doute tirer de ces trois citations l'essentiel du hassidisme: Dieu est partout, il suffit de se livrer à la contemplation; puis, pour se rapprocher de lui, la musique de ses paroles est suffisante, la compréhension savante est tout à fait superflue, et enfin, il n'y a pas plus important que la joie. Ces principes apparaissent dans une formule simple et claire dans le roman Toute une vieque nous (חיים שלמים) allons évoquer en détail par la suite. "Ses mélodies nous nourrissent le matin et le soir " raconte la narratrice du roman au sujet d'un rescapé à moitié juif, «celui qui abrite dans son corps de telles mélodies est un Juif parfait » lui dit - elle, et à un autre elle explique : « une prière, on n'a pas besoin de la comprendre $»^{7}$.

Or, le cadre romanesque et les traits des personnages de l'ouvrage La chambre de Mariana sont tout à fait différents. Mariana est une prostituée ukrainienne chrétienne ; Hugo, qui fait pour elle la lecture des psaumes, est un garçon d'environ treize ans, d'une famille juive assimilée et ils sont, tous les deux, en train d'errer dans les forêts à la fin de la guerre. Mariana sait qu'elle va mourir, car elle était au service de soldats allemands dans une maison close, et c'est justement elle, la belle prostituée 
ukrainienne qui lègue à Hugo, le petit rescapé juif qu'elle a sauvé, l'essentiel de la vraie foi.

9 Il semble que cette conjonction étonnante constitue le noyau dur de l'idée de la religiosité dans l'univers appelfedien. L'acceptation totale du divin dans sa vocation mystique n'a rien à voir ni avec les institutions religieuses, ni avec les appartenances communautaires. Elle correspond sans doute à ce que le Hassidisme considère comme le point intérieur, une lumière qui existe au plus profond de l'âme de chaque être humain créé à l'image. Certes, ce point est caché derrière l'obscurité du mal. Le voyage spirituel qui a comme objectif son dévoilement n'appartient qu'à ceux qui disposent de trois qualités : la sincérité, la générosité et surtout le courage de regarder droit devant lui. Et ces êtres sont des gens simples, Juifs ou non, ouvriers, femmes de ménage, colporteurs et prostituées. Dans son ouvrage autobiographique Histoire d'une vie, Appelfeld parle de trois frères, Otto, porteur de bois, Max, vendeur de charbon et Karl, sourd-muet de naissance. Pour utiliser les mots de l'auteur, ils étaient « courageux et nobles ", de vrais justes ${ }^{8}$.

Revenons à Mariana. Certes, elle n'est pas le seul personnage féminin non-juif qui entretient de forts liens avec le judaïsme dans l'ensemble de l'œuvre d'Appelfeld, mais son histoire est assez particulière dans la mesure où elle fait revivre le mythe chrétien de Marie-Madeleine, la sainte prostituée qui enseigne sa foi aux générations à venir et dont l'oxymore hébraïque "הקדשה הקדושה" (ha-qedesha ha-qedosha, un jeu de mots qui veut dire la prostituée sainte) résume la contradiction. Ce personnage revêt à la fois les caractéristiques biographiques de Marie-Madeleine et les qualités spirituelles du Hassidisme, une combinaison qui se situe par définition au-delà de toute institution et correspond parfaitement à l'idée même de la pureté de la foi la plus profonde. Comme dans la plupart de ses récits, Mariana est inspirée d'une histoire vraie, racontée en détail dans Histoire d'une vie (chapitre 8). Ce dernier récit porte sur une autre Maria, prostituée elle aussi au service des Allemands. Or, le temps passé et l'imaginaire qui se crée avec le recul permettent de tirer de cette histoire vraie, contemporaine de l'errance du petit Aaron Appelfled dans les forêts ukrainiennes des années de la guerre, sa facette spirituelle. Prostituée et alcoolique, belle et charnelle, elle n'incarne qu'une grande âme faite d'amour, de foi et de vérité. Certes, elle enseigne à Hugo les secrets de l'érotisme, mais paradoxalement cet érotisme n'a rien de l'interdit. Au contraire, il constitue l'expression parfaite de l'amour et de la chaleur humaine. Elle abrite son jeune protégé dans son âme et dans son corps, les deux entités créées par Dieu. À la fin $\mathrm{du}$ dixième chapitre, Hugo ose demander à Mariana: «Comment appelle-t-on cet endroit?» Dans la version d'origine, en hébreu, elle lui répond: ma'on|ly , À la différence de la traduction simpliste - «maison close » - le mot ma'on est chargé d'une signification symbolique : non seulement il est rarement utilisé dans ce sens de maison close, mais dans les strates plus classiques de l'hébreu il désigne aussi la maison de Dieu', et encore, surtout dans le langage talmudique et celui de la liturgie médiévale il signifie le ciel ${ }^{10}$. Un seul mot qui englobe en lui « ici-bas » et « là-haut », le terrestre et le céleste.

\section{Les personnages de la mère}

11 Hugo est confié à Mariana par sa mère, une pharmacienne cultivée, juive non pratiquante qui fait confiance à une amie d'enfance. Cette amie est selon ses propres 
mots « une femme de cœur, mais la vie n'a pas été tendre avec elle. ${ }^{11}$ » Comme nous l'avons dit, Mariana n'est pas la seule non-juive à entretenir de liens forts avec le judaïsme tout en remplissant le rôle d'une mère, de substitution ou non.

Nous allons traiter de trois femmes mères, vraies ou substitutives, chrétiennes, juives ou converties au christianisme, qui tout en vivant leur expérience de mère, sont en quête de leur identité religieuse. Chacune d'elles arrive à sa façon à toucher, même si c'est trop tard, ce noyau mystique qui se trouve au-delà des structures existantes.

Le roman Katerina, paru en hébreu en 1989, est le seul parmi les quatre qui soit traduit en français ${ }^{12}$. Puis, שמיון, שלמים חיים, "Toute une vie », paru en 2007, le premier roman dédié par Appelfeld à la mémoire de sa mère Bounia, assassinée en 1941.

Katerina, simple paysanne, chrétienne, retourne dans son village natal d'Ukraine soixante ans après l'avoir quitté par «le chemin des Juifs ${ }^{13}$ » à cause de la bravoure de sa mère, la dureté indifférente de son père, des travaux de la ferme et de la vodka. Elle se remémore sa jeunesse, dans les années précédant la Guerre, du temps où elle servait chez des Juifs. C'est là qu'elle s'ouvrait au monde, cruel et magnifique, et découvrait la chaleur d'un foyer. Elle vit de très près les malheurs des Juifs avant-guerre. Ses patrons sont assassinés lors de pogromes et elle, amoureuse en cachette du maître de la maison Benjamin, nommera ainsi le fils qu'elle aura plus tard d'un vagabond juif. Elle est donc une vraie mère, se considère comme juive et élève son fils ainsi. L'enfant est tué par un gentil ivrogne, qu'elle tue par vengeance, ce qui lui vaudra de passer quarante ans en prison. Elle retrouve sa liberté après-guerre, dans un monde qu'elle croit sans juifs.

15 Katerina est donc une Ukrainienne qui passe sa jeunesse chez les Juifs à s'occuper d'eux, dont le fils assassiné, Benjamin, est à moitié juif, mais elle est aussi une pécheresse, une meurtrière. Malgré cela, son âme trouve la sérénité et la vieille Katerina trouve son point intérieur, elle est en paix avec elle-même et avec son Dieu. En fait, seule, malade et presque aveugle, isolée dans les ruines de la maison parentale, elle est une incarnation parfaite de l'union mystique avec la divinité qui s'exprime à travers la nature, elle passe son temps à contempler :

Maintenant, je sais que c'est la lumière qui m'a ramenée ici. Seigneur, quelle pureté! J'ai parfois envie de tendre la main pour palper la brise qui vient à ma rencontre, douce comme la soie en cette saison.

Le sommeil me fuit en ces claires nuits d'été. J'ai l'impression que c'est un péché de dormir dans une telle clarté. Je comprends enfin ce que signifient les Saintes Écritures. «Il déploie les cieux comme un voile." Jadis, le mot "voile» me paraissait lointain et étrange. Aujourd'hui, je suis capable de le voir. (p. 8)

16 Le milieu juif qui est au centre du roman טמיון («Perdu ») est différent. Il s'agit d'une petite ville autrichienne où la plupart des Juifs sont assimilés et pour des raisons surtout pratiques se convertissent au christianisme en suivant les conseils amicaux du prêtre local. Une autre facette de l'existence juive d'avant-guerre. Après la mort de ses parents, Karl, un fonctionnaire municipal, se convertit afin de mieux faire carrière et soutenir, par sa nouvelle position, les petits commerçants juifs menacés par le pouvoir local.

17 En fait, la conversion ne change rien dans sa vie quotidienne qu'il mène seul dans la maison de ses parents, sans aucun lien avec la tradition d'autrefois. C'est Gloria qui réveille en lui et les souvenirs du temps passé et la sensation de l'amour féminin. Cette paysanne, nourrice et femme de ménage chez sa mère pendant de longues années, qui 
n'était qu'une jeune fille lorsqu'il était enfant, est de retour et prend en charge la maison et son nouveau patron. En fait, c'est la paysanne chrétienne, qui, en suivant les anciennes habitudes de sa patronne morte, prépare le Shabbat, la fête du Nouvel An et surtout, c'est elle qui allume les bougies du souvenir au jour de Grand Pardon à la synagogue. Karl, lui, va se promener à la rivière. Elle est silencieuse et attentive, elle fait son travail quotidien comme une maîtresse de maison juive, elle aime Karl au début comme une mère, puis comme une épouse. Tout comme Mariana dans La chambre de Mariana, Gloria offre à Karl son âme fidèle et son corps chaleureux. Or, il est évident que ce couple n'a pas d'avenir. Karl, Juif converti au christianisme, avec sa bien-aimée, paysanne à l'âme judaïsée, part à la recherche de la nature, à la campagne où il est tué en tant que Juif par de jeunes antisémites. Karl, tout comme ses deux amis d'enfance, convertis eux aussi, vit dans l'obscurité et le mensonge. Il a besoin de Gloria et de sa fidélité silencieuse au monde juif pour dévoiler son appartenance spirituelle et communautaire afin de prendre une autre route, celle qui mène à la nature. Pourtant, la modeste Gloria n'est ni Katerina ni Mariana et Karl n'est ni Hugo l'adolescent ni le petit Benjamin ; il est un adulte qui a trahi ses origines, sa tribu, il reconnaît à peine son tort et trop tard ; même Gloria ne peut rien pour lui. Il a perdu le chemin vers son point intérieur ; il est donc perdu comme l'indique le titre du roman.

18 C'est le même milieu qui est au centre d'un autre roman, Jusqu'à la montée de l'aube עד) publié lui aussi en 1995. Blanca, une juive convertie au יעלה עמוד השחר), christianisme, mariée à un paysan cruel et antisémite, assassine son mari et s'enfuit avec son fils dans la montagne. Tout comme Karl, elle est perdue : elle fait les premiers pas vers la foi, la vraie, mais son voyage, concret et spirituel à la fois, est interrompu brusquement. Meurtrière, elle doit payer sa dette à la société pour devenir plus tard peut-être - nous ne le savons pas - une Katerina d'origine juive....

19 «Ma mère vole et ressemble à un oiseau blanc beaucoup plus qu'à elle-même quand il entend ma voix, ses ailes se mettent à frémir comme un signe de compréhension, mais dès que je tends la main pour le toucher, il s'envole et disparait..$^{14}$ " C'est ainsi que Helga, protagoniste du roman Toute une vieà la recherche de sa mère (חיים שלמים) déportée dans les camps, décrit les apparitions de sa mère dans ses rêves.

20 Dans ce roman, paru en 2007, l'incarnation spirituelle du personnage féminin arrive à la perfection. Helga est la fille unique d'un riche paysan autrichien et d'une mère juive, fine et cultivée, convertie au christianisme par amour. Quand sa mère est déportée, son père l'amène dans un village lointain, chez une tante qui la traite avec hostilité. Dans sa solitude et sa tristesse, Helga s'enfuit et entreprend un long et difficile voyage à la recherche de sa mère. C'est un voyage à double sens : concrètement, elle traverse le pays, elle passe un moment dans une chapelle, elle frappe aux portails des camps en route et enfin elle arrive à s'intégrer dans l'un d'eux où elle pense trouver sa mère. En effet, elle y était, mais tombée malade, elle avait été évacuée - lui apprennent les prisonnières à bout de forces. La guerre est presque finie ; les alliés bombardent tout autour ; Helga, très affaiblie, arrive malgré tout à s'enfuir puis continue à errer d'un centre médical à un autre à la recherche de sa mère. Manfred, un vieux paysan, l'homme à tout faire de la ferme de son père, qui est en réalité un tzadik nistar, un juste caché, la trouve. Il lui annonce la mort de son père lors des bombardements puis amène le corps de Gisela, la mère, qui vient de mourir dans un hôpital militaire américain. Après la semaine de deuil et la fermeture du centre d'accueil, Helga part pour la Palestine. 
21 Or, l'essentiel du roman n'est pas dans ce récit douloureux et tragique raconté par une voix tendre et précise. La vraie histoire porte sur le voyage interne, la quête spirituelle, qui englobe l'action par un dialogue incessant entre Helga et sa mère, soit à travers les rêves, soit par des hallucinations. Le vécu réel n'a presque pas de poids par rapport au vécu imaginaire qui s'achève au moment où Helga, l'adolescente devenue une jeune femme, intègre en elle le personnage de sa mère, son ange gardien. En fait, elle devient sa mère et ainsi continue à vivre.

S'agit-il d'une expérience vécue par Appelfeld lui-même au cours de ses années d'errance, enfant, seul dans les forêts? Il le dit, et à plusieurs reprises. "Le désir intense que j'avais de mes parents fit que je les transportais là où je me cachais, et je parlais avec eux comme s'ils étaient assis à côté de moi. Ces conversations me procuraient une sorte de joie confiante, et, par-dessus tout, le sentiment que je n'étais pas seul au monde. D'autres enfants m'ont dit après la guerre qu'ils avaient connu ce sentiment, eux aussi, dans le bois ${ }^{15}$. "

23 À travers l'image de sa mère, Helga trouve son point intérieur, elle arrive à vivre une spiritualité parfaite qui est semée en elle peu à peu, tout au long de son voyage. Le chemin vers elle-même est constitué de trois étapes. Chacune est incarnée par une femme qui symbolise dans sa façon d'être la religiosité pure, au-dessus de toute appartenance institutionnelle. La première est Theresa, la religieuse qui enseignait à l'école dans le village perdu où Helga se cachait après la déportation de sa mère. Contrairement à la tante, une paysanne antisémite, Theresa lui procure un nid d'amour et de compréhension :

Après peu de temps, je lui ai dit que maman était juive. Elle m'a regardée et a dit : « tu peux être fière" sans ajouter quoi que ce soit. Tout de suite je sus que je pouvais partager mon secret avec elle. Le lendemain, nous avons parlé de la méchanceté et des gens qui avaient perdu l'image de Dieu, comme Caïn. J'ai demandé ce que voulait dire ce mot, image.

"C'est un cadeau offert à l'homme par Dieu. Un cadeau précieux. Lorsque l'homme le garde, il est lié à Dieu ; celui qui détruit l'image appartient aux Sodomites. »

- La nuit j'ai entendu maman me parler: merci Dieu de nous avoir empêchés de nous comporter comme eux et de nous avoir laissé l'image. Je prie Dieu de me donner la force de garder l'image même dans le camp ${ }^{16}$.

C'est Theresa qui confirme à la jeune fille sa judaïcité :

Suis-je juive?

En effet, tu es juive. Si ta mère est juive, tu es juive ${ }^{17}$.

24 L'étape suivante est la rencontre avec Elsa, la vieille tante aveugle de Theresa qui habite dans une petite hutte isolée à côté de la chapelle Saint-Paul. Il semble que plus le mal s'approche de Helga dans ses recherches concrètes de sa mère, plus c'est elle qui s'approche du divin dans son voyage interne. Elle le fait par le biais d'Elsa l'aveugle, une transformation tardive de la vieille Katerina. Elsa, avec sa petite cabane, son jardin et sa fenêtre toujours ouverte, Elsa la végétarienne tout comme Gisela, la mère, Elsa qui passe sa vie dans la contemplation la plus intime et dont l'existence physique et sociale n'a presque pas de traces. En fait, Elsa est à mi-chemin entre la vie d'ici-bas et la vie de là-haut. Sa façon d'être constitue l'étape préparatoire de la rencontre d'Helga avec sa mère morte qui est transformée au cours des dernières phases de sa vie en un ange.

25 En effet, dès que Helga arrive, de son propre gré, à pénétrer dans le camp et à devenir une prisonnière comme les autres, la facette angélique de sa mère devient de plus en plus présente. Non seulement elle est l'ange gardien de sa fille dans son imaginaire, mais c'est ainsi qu'elle est aperçue par les femmes qui l'ont rencontrée dans les 
différents camps. Les bribes de descriptions que Helga tire d'elles, sont toujours exprimées par le langage du sacré :

Gisela était notre lumière. Nous ne savions pas conserver notre âme, disait-elle, ce n'est pas par hasard si nous avons oublié la prière ${ }^{18}$.

Et puis :

La nuit, elle nous parlait. Elle était, tu le sais mieux que moi, très réservée et parlait à peine. Mais les nuits, elle se réveillait et nous parlait comme si elle était un ange de Dieu, je dis un ange de Dieu, car je ne trouve pas d'autres mots. Elle avait des mots simples, sans manière aucune, et les phrases surgissaient sans cesse, comme celle-ci que je n'oublierai jamais: «malgré tout, il est formidable d'être juif » et la nuit suivante : « il faut aimer les torturés, ils

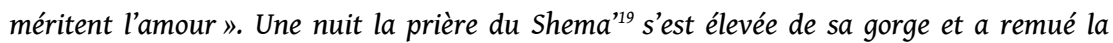
cabane. Je ne sais pas de quel monde Gisela est venue chez nous ${ }^{20}$.

Dans le centre médical pour les survivants où elle travaille à la fin de la guerre, Helga apprend l'hébreu et le yiddish, les deux langues sacrées selon ses mots. Elle apprend aussi à chanter dans ces langues, des chants populaires en yiddish tout comme la liturgie ou des chansons sionistes en hébreu, comme si inconsciemment, par un fil mystérieux, elle avait entendu les dernières paroles de sa mère telles qu'elles sont rapportées par une de rescapées :

Gisela était notre lumière. Elle nous a enseigné des mots en hébreu et en yiddish. Après une journée difficile ou une punition collective, elle nous a récité avec beaucoup d'émotion la prière du Shema'. Sais-tu de quoi il s'agit? Sais-tu quelle puissance il faut pour se frayer un chemin dans les cours des gens désespérés ${ }^{21}$ ?

Ce sont des chansons en yiddish et en hébreu qui accompagnent Gisela lors de son enterrement dans les ruines de l'ancien cimetière juif à proximité de la ville. Elle se montre à sa fille une dernière fois avant son départ pour la Palestine, et ses mots scellent à jamais leur lien mystique : « La mort n'est qu'une illusion. Nous sommes liées l'une à l'autre par un amour très fort ${ }^{22} »$.

\section{BIBLIOGRAPHIE}

APPELFELD, Aharon (1989), קאטרינה (Katerina), Keter.

APPELFELD, Aharon (2004), Histoire d'une vie, L'olivier. (Version originale parue en 1999, Keter.

APPELFELD, Aharon (2006a), « Jusqu'à présent j'ai écrit le premier tiers... - entretien avec Aharon Appelfeld » in « La Shoah dans la littérature israélienne », Revue de l'histoire de la Shoah, $\mathrm{n}^{\circ} 184$.

APPELFELD, Aharon (2006b), L'héritage nu, L'olivier.

APPELFELD, Aharon (2007), שלמיםחיים(Toute une vie), Keter.

APPELFELD, Aharon (2008), La chambre de Mariana, L'olivier, Paris. (Version originale parue en 2006, Keter, Jérusalem, sous le titre פרחי האפלה).

BAUMGARTEN, Jean (2006), La naissance du Hassidisme, Mystique, rituel et société, Albin Michel, Paris.

BUBER, Martin (1993), La légende du Baal Shem Tov, Le Rocher. 
KASPI, Zehava (2005), « לקלף את הקליפות ולחשוף את הגרעין ( Éplucher la peau, dévoiler le noyau »), in The World of Appelfeld, Selection of Essays, Beer Sheva.

MILNER, Iris (2005), « בשם האם, טראומה של אבדן ופרובלמטיקה של זהות («Au nom de la mère etc. »), in The World of Appelfeld, Selection of Essays, Beer Sheva.

\section{NOTES}

1. Voir à titre d'exemple: Milner (2005), pp. 35-47; Kaspi (2005), pp. 47-57.

2. Voir : entretien avec Aharon Appelfeld (2006a), pp. 80-81.

3. À signaler en particulier celui de Wischnitza dont on va parler par la suite.

4. Paris 1993, voir: Baumgarten (2006).

5. La version française est parue en 2008, Paris, L'olivier, p. 265 ; l'original en hébreu - en 2006, Keter, Jérusalem.

6. Pour en savoir plus voir : Buber (1993), p. 272.

7. Voir : Appelfeld (2007), pp. 222 et 226.

8. Voir : Appelfeld (2004), chapitre 10.

9. Voir : Ps 26:8 ; Jer 25:30, etc.

10. Hagiga 12 : et chez El'azar Ha-Kalir et Solomon Ibn Gabirol.

11. Voir : Appelfeld (2008), p. 119

12. Chez Points-Seuil, 1996 et 2007.

13. C'est ainsi que la route à la sortie du village est appelée par les paysans.

14. Voir Appelfeld (2007), p. 66, la traduction est la mienne. À signaler que l'oiseau en hébreu est un mot féminin.

15. Voir : Appelfeld (2006b), p. 87.

16. Voir : Appelfeld (2007), pp. 68-69.

17. Ibid.p. 100.

18. Ibid.p. 165.

19. שמע קריאת.

20. Ibid. pp. 188-189.

21. Ibid. p. 200.

22. Ibid. p. 253.

\section{RÉSUMÉS}

L'objet de cet article est le concept de religiosité dans la narration d'Appelfeld et ses relations avec le hassidisme.

Cette question est examinée principalement sur la base de trois de ses romans: Katerina, La chambre de Mariana et Toute une vie, en portant une attention particulière aux protagonistes féminins, juifs aussi bien que catholiques. Selon l'article, Appelfeld a créé un nouveau type de judaïsme, primitif, qui est totalement déconnecté de toute institution sociale ou religieuse. Cette « nouvelle » religion dont la nature est quelque peu mystique, est essentiellement basée sur l'idée de panthéisme, d'une part et à la contemplation de l'autre et à partir de ces points de vue, il est 
assez lié aux principales caractéristiques du hassidisme, même si les non-juifs pourraient également prendre part à cette expérience très spéciale.

The subject matter of the article is the concept of religiosity as figures in Appelfeld's narration and it's relationships with Hassidism.

This question is examined mostly on the basis of three of his novels: Katerina, Flowers of Darkness and A Perfect Life, with extensions to few more, on paying a special attention to feminine protagonists, Jewish as well as Catholics. According to the article, Appelfeld has created a new type of Judaism, a primitive one, which is totally disconnected of any social or religious institution. This "new" religion whose nature is somewhat mystical, is mainly based on the idea of pantheism on one hand and contemplation on the other and from these points of view, it is quite related to the main characteristics of the Hassidism even if non-Jewish might also take part in it's very special experience.

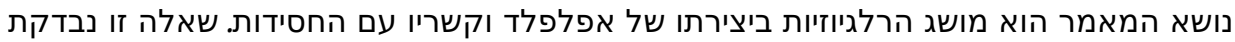

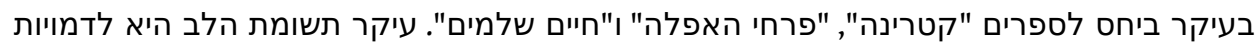

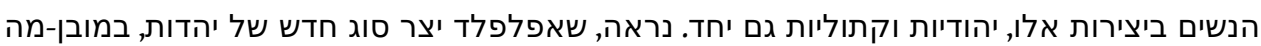

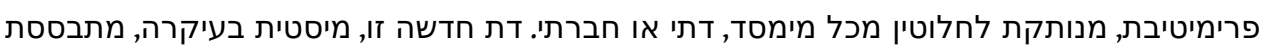

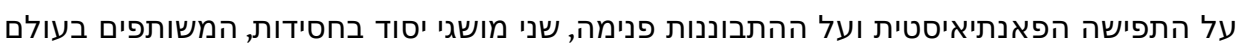
של אפלפלד גם לדמויות שאינן יהודיות.

\section{INDEX}

Index chronologique : vingtième siècle

Thèmes : sciences religieuses, littérature

\section{מילות מפתח}

אהרון אפלפלד, החסידות, דתיות, נשים, הרומן תווים, דתיים ללימודים, ספרות, המאה:

העשרים

Mots-clés : hassidisme, religiosité, femmes, personnages de roman, Appelfeld Aharon (1932-)

Keywords : hasidism, religiosity, women, novel characters, literature, Appelfeld Aharon (1932-), twentieth century 